\title{
Deep learning for cosmology
}

\section{Celia Escamilla-Rivera*}

Instituto de Ciencias Nucleares, Universidad Nacional Autónoma de México, Circuito Exterior C.U., A.P. 70-543, México D.F. 04510, México.

E-mail: celia.escamilla@nucleares.unam.mx

In this paper we will describe ongoing efforts to shed light on still-unanswered questions in fundamental physics using cosmological observations. We will explain how we can use measurements of the Supernovae data and Baryon Acoustic Oscillations to reconstruct the detailed physics of the dark universe. Also it will address this inverse-problem reconstruction from a Bayesian and Deep Learning perspectives.

Artificial Intelligence for Science, Industry and Society, AISIS2019

October 21-25, 2019

Universidad Nacional Autónoma de México, Mexico City, México

${ }^{*}$ Speaker. 


\section{Introduction}

A highlight in observational cosmology is the origin and nature of the cosmic accelerated expansion. The standard cosmological model that is consistent with current cosmological observations is the so-called concordance model or $\Lambda \mathrm{CDM}$. According to this scenario, the observed accelerating expansion is related to the repulsive gravitational force of a Cosmological Constant $\Lambda$ with constant energy density $\rho$ and negative pressure $p$. This proposal has been the backbone of the standard cosmology since the nineties, but simple enough as it is the proposal has a couple of theoretical problems, two of them are the fine tuning argument and coincidence problem $[1,2]$. In order to solve or at least relax these problems, some proposals have lead to alternative scenarios that can modify the General Relativity (GR) or consider a landscape with a dynamical dark energy. Is in this way that dark energy emerge as a cosmological solution since it can be described as a fluid parametrised by an equation of state (EoS), which can be written in terms of the redshift, $w(z)$. So far, the properties of this EoS remains under-researched. Just to mention a few, there are a zoo of proposals on dark energy parameterisations discussed in the literature (see, e.g., $[3,77,9,50,5,6,7])$, addresing from parameterisation as Taylor-like series to dynamical $w(z)$ that can provide oscillatory behaviours.

Nowdays, the techniques to discriminate between models and confront them with $\Lambda \mathrm{CDM}$, are based in the calculations of the constraints on the EoS free parameter(s) of the models. This methodology has been done using observables that can show the cosmic acceleration such as: supernovae type IA (SNeIa), baryon acoustic oscillations (BAO), cosmic microwave background (CMB), weak lensing spectrum, etcetera. The relevance of using these observations is due to the precision with which dark energy can be probed. Currently, some measurements such as the Pantheon from supernovae [83], BOSS [12], just to cite a few, point out a way to constrain these EoS parameters. These observations allow deviations from the $\Lambda$ CDM model, which are usually parametrised by the free EoS parameters. In past years there have been many observations related to the verification of the cosmic acceleration, e.g from Union $2.1^{1}$ to the Joint LightCurve Analysis $[10,84]$. But the statistics has been improved due the density of data this kind of supernovae.

\section{How to model dark energy}

One of the first steps to understand the behaviour of the cosmic acceleration remains in that we require an energy density with negative pressure at late times. To achived this we need to express the ratio between the pressure and energy density as negative, i.e., $w(z)=P / \rho<0$. In order to develop the evolution equations for a universe with this kind of fluid, we start by introducing in Einstein equations a Friedmann-Lemaitre-Robertson-Walker metric to obtain the Friedmann and Raychaudhuri equations for a spatially flat universe

$$
E(z)^{2}=\left(\frac{H(z)}{H_{0}}\right)^{2}=\frac{8 \pi G}{3}\left(\rho_{m}+\rho_{D E}\right)\left[\Omega_{0 m}(1+z)^{3}+\Omega_{0(D E)} f(z)\right]
$$

and

$$
\frac{\ddot{a}}{a}=-\frac{H^{2}}{2}\left[\Omega_{m}+\Omega_{D E}(1+3 w)\right]
$$

\footnotetext{
${ }_{1}$ http://supernova.lbl.gov/Union/
} 
where $H(z)$ is the Hubble parameter in terms of the redshift $z, G$ the gravitational constant and the subindex 0 indicates the present-day values for the Hubble parameter and matter densities.

From (2.2) it is possible to obtain the energy conservation equation, in that way the energy density of the non-relativistic matter is $\rho_{m}(z)=\rho_{0 m}(1+z)^{3}$. And the $\rho_{m}$ is given by:

$$
\rho_{m}(z)=\rho_{0 m}(1+z)^{3},
$$

and the dark energy density can be model as $\rho_{D E}(z)=\rho_{0(D E)} f(z)$, where can be written as:

$$
\rho_{D E}(z)=\rho_{0(D E)} f(z) .
$$

If we assuming that the energy-momentum tensor (on the right side of the Einstein's equations) $T^{\mu v}$ is a perfect fluid (without viscosity or stress effects), i.e. $\nabla_{\mu} T^{\mu v}=0$, the form of $f(z)$ can be restricted to be:

$$
f(z)=e^{\left[3 \int_{0}^{z} \frac{1+w(z)}{1+z} d z\right]} .
$$

Now, the behaviour of the latter is restricted directly to the form of $w(z)$, which can give a description of the Hubble function (which can be normalised by the constant Hubble $H_{0}$ ), as e.g., in the case of quiessence models ( $w=$ const.) the solution of $f(z)$ is $f(z)=(1+z)^{3(1+w)}$. If we consider the case of the cosmological constant $(w=-1)$ then $f=1$.

Some interesting insights of the above forms for $w(z)$ has been reported in $[77,78]$ and references therein, where a dark energy density $\rho_{D E}$ with varying and non-varying $w(z)$ are considered.

\section{Estimating the cosmological parameters}

After we have defined a specific cosmological model, we can then perform their test using astrophysical observations. The methodology can be described by a simple calculation of the usual $\chi^{2}$ method and then process the MCMC chains computational runs around a certain value (observational(s) point(s)), and obtain the best fit parameter(s) of this process. Parameter estimation is usually done by computing the so-called likelihood function for several values of the cosmological parameters. For each data points in the parameter space, the likelihood $\mathscr{L}$ function gives the minimise probability of obtaining the observational data that was obtained if the hypothesis parameters had the given values (or priors). For example, the standard cosmological model $\Lambda \mathrm{CDM}$ is described by six parameters, which include the amount of dark matter and dark energy in the universe as well as its expansion rate $H$. Using the CMB data (which is the accuracy data that we understand very well so far), a likelihood function can be constructed. The information given by $\mathscr{L}$ can tell which values of these parameters are more likely, i.e by probing many different values. Therefore, we are able to determine the values of the parameters and their uncertainties via error propagation over the free parameters of the model.

Now, the following question is what kind of astrophysical surveys ${ }^{2}$ can we use to test the cosmological models. In the next sections we described the most used surveys that are employed to analise the cosmic acceleration. Is important to mention that these surveys spread depending its

\footnotetext{
${ }^{2}$ This word in the coloquial language also can be replaced by likelihood -do not misunderstood with the function $\mathscr{L}$. Or simple we can called as samplers.
} 
own nature. We have three types of observations classified as: standard candels (e.g supernovae -properly calibrated-, which characteristic function is the luminosity distance), standard rulers (e.g BAO, which characteristic function is the angular/volumen distance) and the standard sirens (e.g gravitational waves, which can be described by frequencies or chirp masses depending the observation). The set of all of them can describe a precise statistics, but by separate, each of them have intrinsic problems due their physical definition. For supernovae, the luminosity distance have in their definition an integral of the cosmological model, therefore when we perform the error propagation, the uncertainty is high. This disadvantage can be compensated by the large population of data points in the sampler. On the other hand, the uncertainty is less for standard rulers in comparison to supernovae. For this case, the definition of angular distance does not include integrals. The price that we pay in order to use this kind of sampler is that the population of data is very small (e.g from surveys like BOSS or CMASS, we have only 7 data points). Moving forward, the observation of gravitational wave standard sirens would be developed into a powerful new cosmological test due that they can play an important role in breaking parameter degeneracies formed by other observations as the ones mentioned. Therefore, gravitational wave standard sirens are of great importance for the future accurate measurement of cosmological parameters. In this part of the chapter, we are going only to develop the use of the first two kind of observations.

\section{Supernovae sampler}

Along the ninety years, since their discovery, Type Ia supernovae (SNIa) have been the proof of the current cosmic acceleration. The surveys have been changing given us a large population of observations, from Union $2.1^{3}$ to the Joint LightCurve Analysis [10, 84], the data sets have been incrementing observations and also their redshift range. Currently, the Pantheon sampler, which consist of a total 1048 Type Ia supernovae (SNIa) in 40 bins [83] compressed, is the largest spectroscopically confirmed SNIa sample to date. This latter characteristic makes this sample attractive to constrain with considerably precision the free cosmological parameters of a specific model.

SNIa can give determinations of the distance modulus $\mu$, whose theoretical prediction is related to the luminosity distance $d_{L}$ according to:

$$
\mu(z)=5 \log \left[\frac{d_{L}(z)}{1 M p c}\right]+25
$$

where the luminosity distance is given in units of Mpc. In the standard statistical analysis, one adds to the distance modulus the nuisance parameter $\mu_{0}$, an unknown offset sum of the supernovae absolute magnitude (and other possible systematics), which is degenerate with $H_{0}$.

Now, the statistical analysis of the this sample rests on the definition of the modulus distance as:

$$
\mu\left(z_{j}, \mu_{0}\right)=5 \log _{10}\left[d_{L}\left(z_{j}, \Omega_{m} ; \theta\right)\right]+\mu_{0},
$$

where $d_{L}\left(z_{j}, \Omega_{m} ; \theta\right)$ is the Hubble free luminosity distance:

$$
d_{L}\left(z, \Omega_{m} ; \theta\right)=(1+z) \int_{0}^{z} \mathrm{~d} z^{\prime} \frac{1}{E\left(z^{\prime}, \Omega_{m} ; \theta\right)} .
$$

\footnotetext{
${ }^{3}$ http://supernova.lbl.gov/Union/
} 
With this notation we expose the different roles of the several cosmological parameters appearing in the equations: the matter density parameter $\Omega_{m}$ appears separated as it is assumed to be fixed to a prior value, while $\theta$ is the EoS parameters $w_{i}$. These later are the parameters that we will be constraining by the data. The best fits will be obtained by minimising the quantity

$$
\chi_{S N}^{2}\left(\mu_{0}, \theta\right)=\sum_{j=1}^{N} \frac{\left.\left(\mu\left(z_{j}, \Omega_{m} ; \mu_{0}, \theta\right)\right\}-\mu_{o b s}\left(z_{j}\right)\right)^{2}}{\sigma_{\mu, j}^{2}}
$$

where the $\sigma_{\mu, j}^{2}$ are the measurement variances. And nuisance parameter $\mu_{0}$ encodes the Hubble parameter and the absolute magnitude $M$, and has to be marginalised over.

From now on, we will assume spatial flatness, therefore the luminosity distance is related to the comoving distance $D$ via the equation

$$
d_{L}(z)=\frac{c}{H_{0}}(1+z) D(z)
$$

where $c$ is the speed of light, so that, using (4.1) we can obtain

$$
D(z)=\frac{H_{0}}{c}(1+z)^{-1} 10^{\frac{\mu(z)}{5}-5} .
$$

The normalised Hubble function $E(z)$ can be obtained by taking the inverse of the derivative of $D(z)$ with respect to the redshift $D(z)=\int_{0}^{z} H_{0} d \tilde{z} / H(\tilde{z})$. A usual alternative, instead of using the full set of parameters for this sampler, is to use the Pantheon plugin for CosmoMC to constrains cosmological models (something similar as in the case of Joint Light Curve Analysis sampler [84]).

Since we are taking nuisance parameter $M$ in the sample, we choose the respective values of $\mu_{0}$ from a statistical analysis of the $\Lambda \mathrm{CDM}$ model with Pantheon sample obtained by fixing $H_{0}$ to the Planck value given in [53]. Is common to perform this kind of fit using computational tools that can run a standard MCMC chains. In cosmology -at least at the moment this paper is writingseveral codes have been implemented in order to perform the statistical fit of this parameter. The lector can explore the tool called MontePython code ${ }^{4}$ and run a standard MCMC for $M$ using the model of their preference. As an example, if we run a $\Lambda \mathrm{CDM}$ model with this supernovae sample, the mean value obtained will be $\mu_{0}=-19.63$.

\section{Baryon Acoustic Oscillation sampler}

As a standard rulers, these astrophysical observations can contribute important features by comparing the data of the sound horizon today to the sound horizon at the time of recombination (extracted from the CMB anisotropy data). Usually, the baryon acoustic distances are given as a combination of the angular scale and the redshift separation.

To define these quantities we require a relationship via the ratio:

$$
d_{z} \equiv \frac{r_{s}\left(z_{d}\right)}{D_{V}(z)}, \quad r_{s}\left(z_{d}\right)=\frac{c}{H_{0}} \int_{z_{d}}^{\infty} \frac{c_{s}(z)}{E(z)} \mathrm{d} z
$$

\footnotetext{
${ }^{4}$ https://monte-python.readthedocs.io/en/latest/
} 
where $r_{s}\left(z_{d}\right)$ is the comoving sound horizon at the baryon dragging epoch,

$$
r_{s}\left(z_{d}\right)=\frac{c}{H_{0}} \int_{z_{d}}^{\infty} \frac{c_{s}(z)}{E(z)} \mathrm{d} z
$$

and $z_{d}$ is the drag epoch redshift with $c_{s}^{2}=c^{2} / 3\left[1+\left(3 \Omega_{b 0} / 4 \Omega_{\gamma 0}\right)(1+z)^{-1}\right]$ as the sound speed with $\Omega_{b 0}$ and $\Omega_{\gamma 0}$, which are the present values of baryon and photon parameters, respectively.

We define the dilation scale as

$$
D_{V}\left(z, \Omega_{m} ; w_{0}, w_{1}\right)=\left[(1+z)^{2} D_{A}^{2} \frac{c z}{H\left(z, \Omega_{m} ; w_{0}, w_{1}\right)}\right]^{1 / 3},
$$

where $D_{A}$ is the angular diameter distance given by

$$
D_{A}\left(z, \Omega_{m} ; w_{0}, w_{1}\right)=\frac{1}{1+z} \int_{0}^{z} \frac{c \mathrm{~d} \tilde{z}}{H\left(\tilde{z}, \Omega_{m} ; w_{0}, w_{1}\right)} .
$$

Using the comoving sound horizon, we can relate the distance ratio $d_{z}$ with the expansion parameter $h$ (defined such that $H \doteq 100 h$ ) and the physical densities $\Omega_{m}$ and $\Omega_{b}$. Therefore, we have

$$
r_{s}\left(z_{d}\right)=153.5\left(\frac{\Omega_{b} h^{2}}{0.02273}\right)^{-0.134}\left(\frac{\Omega_{m} h^{2}}{0.1326}\right)^{-0.255} \mathrm{Mpc},
$$

with $\Omega_{m}=0.295 \pm 0.304$ and $\Omega_{b}=0.045 \pm 0.00054$ [84]. As we mentioned above, unfortunately so far we have a very low data population of this sampler. Moreover, as an example for this text, we employed compilations of three current surveys: $d_{z}(z=0.106)=0.336 \pm 0.015$ from 6-degree Field Galaxy Survey (6dFGS) [25], $d_{z}(z=0.35)=0.1126 \pm 0.0022$ from Sloan Digital Sky Survey (SDSS) [26] and $d_{z}(z=0.57)=0.0726 \pm 0.0007$ from Baryon Oscillation Spectroscopic Survey (BOSS) with high-redshift CMASS [27].

We can also, add to the full sample three correlated measurements of $d_{z}(z=0.44)=0.073$, $d_{z}(z=0.6)=0.0726$ and $d_{z}(z=0.73)=0.0592$ from the WiggleZ survey [28], which has the inverse covariance matrix:

$$
\mathbf{C}_{\mathbf{W i g g l e Z}}^{-\mathbf{1}}=\left(\begin{array}{ccc}
1040.3 & -807.5 & 336.8 \\
-807.5 & 3720.3 & -1551.9 \\
336.8 & -1551.9 & 2914.9
\end{array}\right)
$$

In order to perform the $\chi^{2}$-statistic, we define the proper $\chi^{2}$ function for the BAO data as

$$
\chi_{\mathrm{BAO}}^{2}(\theta)=\mathbf{X}_{\mathrm{BAO}}^{T} \mathbf{C}_{\mathrm{BAO}}^{-1} \mathbf{X}_{\mathrm{BAO}}
$$

where $\mathbf{X}_{\mathbf{B A O}}$ is given as

$$
\left.\mathbf{X}_{\mathbf{B A O}}=\left(\frac{r_{s}\left(z_{d}\right)}{D_{V}\left(z, \Omega_{m} ; w_{0}, w_{1}\right)}\right)-d_{z}(z)\right)
$$

Then, the total $\chi_{\mathrm{BAO}}^{2}$ is directly obtained by the sum of the individual quantity by using (5.7) in

$$
\chi_{\mathrm{BAO}-\mathrm{total}}^{2}=\chi_{\mathrm{6 \textrm {dFGS }}}^{2}+\chi_{\mathrm{SDSS}}^{2}+\chi_{\mathrm{BOSSCMASS}}^{2}+\chi_{\mathrm{WiggleZ}}^{2} .
$$




\section{About deep learning in cosmology}

Although, Bayesian evidence remains the preferred method compared with information criterions and gaussian processes on the literature, a complete Bayesian inference for model selection -this to have an scenario where we can discriminate a pivot model from a hypothesis- is very computationally expensive and often suffers from multi-modal posteriors and parameter degeneracies. As we pointed out in the later section, the calculation of the evidence leads to a large time consumption to obtain the final result.

As the study of the Large Scale Structure (LSS) of the universe indicates, all our knowledge relies on state-of-the art cosmological simulations to address a number of questions by constraining the cosmological parameters at hand using Bayesian techniques. Moreover, due to the computational complexity of these simulations, some studies look remain computationally infeasible for the forseeable future. Is at this point where computational techniques as machine learning can have a number of important uses, even for trying to understand our universe.

The idea behind the machine learning is based in consider a neural network with a complex combination of neurons organised in nested layers. Each of these neuron implements a function that is parametrised by a set of weights $W$. And every layer of a neural network thus transforms one input vector -or tensor depending the dimension- to another through a differentiable function. Theoretically, given a neuron $n$ it will receive an input vector and the choice of an activation function $A_{n}$, the output of the neuron can be computed as

$$
\begin{aligned}
h^{<t>}= & A_{n}\left(W_{h} \cdot h^{<t-1>} \cdot+W_{x} \cdot x^{<t>}+b_{a}\right), \\
& y^{<t>}=A_{n}\left(W_{y} \cdot h^{<t>}+b_{y}\right),
\end{aligned}
$$

where $h^{<t>}$ is called the hidden state, $A_{n}$ is the activation function and $y^{t}$ is the output.

The goal in to introduce a set of data in order to train this array and therefore the architecture can learn to finally give an output set of data. For example: the network can learn the distribution of the distance moduli in the dark energy models, then feed the astrophysical samplers (surveys) to the network to reconstruct the dark energy model and then discriminate the most probable model. 5

Moreover, while neural networks can learn complex nested representations of the data, allowing them to achieve impressive performance results, it also limits our understanding of the model learned by the network itself. The choice of an architecture [85] can have an important influence on the performance of the neural network. Some design have to made concerning the number and the type of layers, as well as the number and the size of the filters used in each layer. A convenient way to select these choices is typically through experimentation -which for our universe, we will need these to happen first.- As it is, we can select the size of the network, which depends on the number of training test as networks with a large number of cosmological parameters are likely to overfit if not enough training test are available.

At the moment these lines are writing, a strong interest over this kind of algorithm is bringing new opportunities for data-driven cosmological discovery, but will also present new challenges for

\footnotetext{
${ }^{5}$ In this paper we are employing a Recurrent Neural Network. There are several in this machine learning field e.g. in [85] and references therein.
} 
adopting machine learning -or, in our case, a subset of this field, deep learning- methodologies and understanding the results when the data are too complex for traditional model development and fitting with statistics. A few proposals in this area has been done to explote the deep learning methods for measurements of cosmological parameters from density fields [86] and for future large-scale photometric surveys [71].

\section{Deep learning for dark energy}

A first target in order to start to train an astrophysical survey is to design an architecture with an objective function of neural networks that can have many unstable points and a local minima. This architecture makes the optimisation process very difficult, but in real scenarios, high levels of noise degrade the training data and typically results in optimisation scenarios with more local minima and therefore increases the difficulty in training the neural network. It can thus be desirable to start optimising the neural network using noise-free data which typically yield smoother scenarios. As an example, in Figure 1 we present a standard network using an image of a cosmological simulation (the data) and then divided an array of several layers to finally extract the output cosmological parameters value. Each neuron use a Bayesian process to compute the error propagation as it is done in the standard inference analyses.

We can describe a quickly, but effective, recipe to develop a Recurrent Neural Network with a Bayesian computation training in the following steps:

- Step 1. Construction of the the neural network. For a Recurrent Neural Network method we can choose values that have one layer and a certain number of neurons (e.g you can start with 100 for a supernovae sampler).

- Step 2. Organising the data. We need to sort the sampler from lower to higher redshift in the observations. Afterwards, we re-arrange our data using the number of steps (e.g try with 4 steps numbered as $x_{i}$ for a supernovae sampler).

- Step 3. Computing the Bayesian training. Due to the easiness of neural networks to overfit, it is important to choose a mode of regularisation. With a Bayesian standard method to compute the evidence, the algorithm can calculate errors via regularisation methods[89]. Finally, over the cost function we can use Adam optimiser.

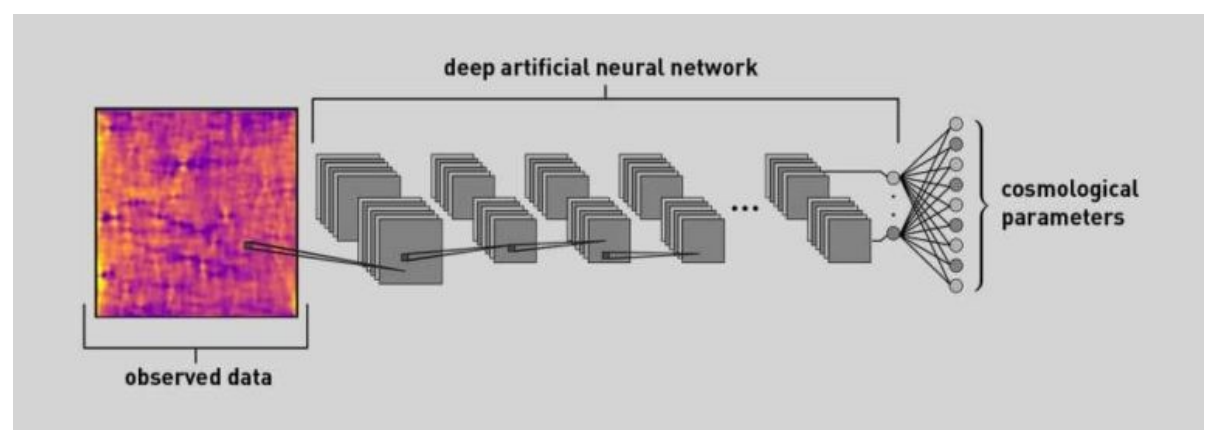

Figure 1: A deep learning architecture for cosmology, 
- Step 4. Training the entire architecture. It is suitable to consider a high number of epochs (e.g for a sampler as Pantheon, you can try with 1000 epoch per layer). After the training, it is necessarily to read the model and apply more times the same dropout to the initial model. The result of this step is the construction of the confidence regions.

- Step 5. Computing modulus distance $\mu(z)$ for each cosmological model. Using the definitions of $E(z)$, we can compute $\mu(z)$ by using a specific dark energy equation of state in terms of $z$ and then integrating them.

- Step 6. Computing the best fits. Finally, the output values can be obtained by using the training data as a simulated sample. We use the publicly codes CLASS ${ }^{6}$ and Monte Python ${ }^{7}$ to constrain the models as it is standard for usual Bayesian cosmology.

The results of this recipe can be seeing in Figure 2.

\section{Conclusions}

In this paper we discuss how to derive the equations of state for a specific dark energy model. Also we studied the standard models of dark energy in order to project the cosmic acceleration according to the current data available in the literature. Is important to remark that each Bayesian statistics performed will depend solely on the data used to developed them. More the data, better

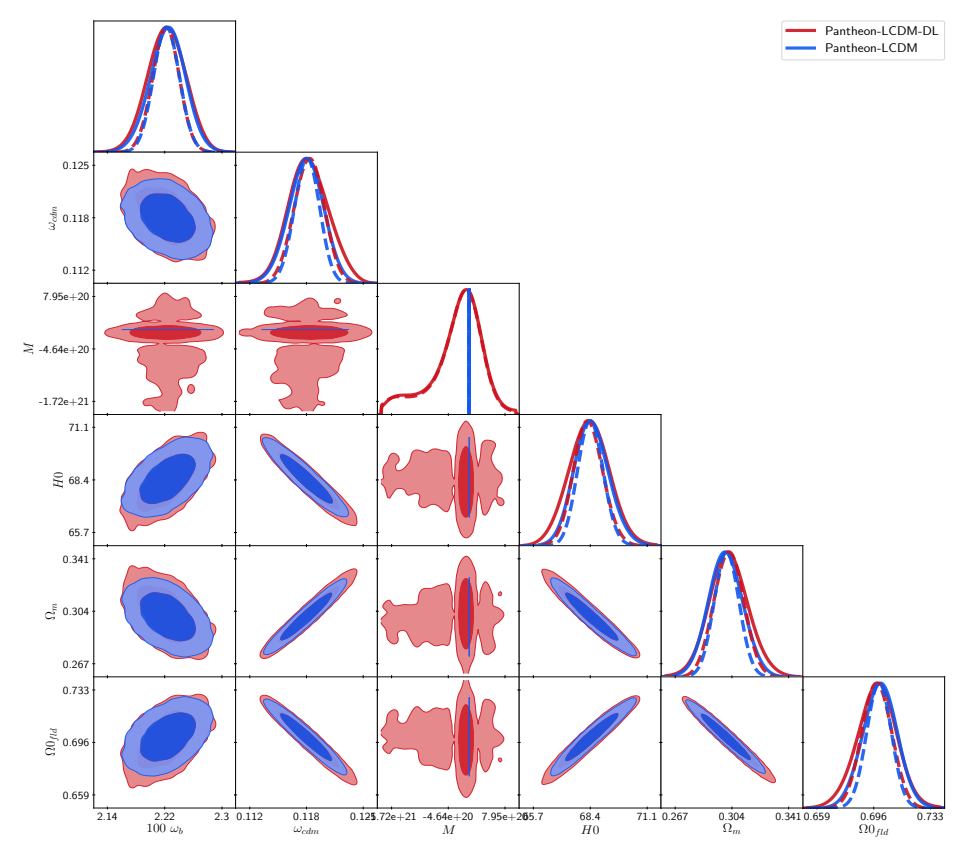

Figure 2: Statistical contours levels for $\Lambda \mathrm{CDM}$ using observational data (red color) and training deep learning data (blue color)

\footnotetext{
${ }^{6}$ https://github.com/lesgourg/class_public

${ }^{7}$ https://github.com/baudren/montepython_public
} 
the statistics. So we expect that future surveys will improve the constrains over the cosmological parameters, not only at background level, but also at perturbative level.

The exploration of these astrophysical surveys have reached a new scenario in regards to the machine learning techniques. These kind of techniques allow to explore -without technical problems in the astrophysical devices- scenarios where the pivot model of cosmology, $\Lambda \mathrm{CDM}$, a theoretical framework that accurately describes a large variety of cosmological observables, from the temperature anisotropies of the cosmic microwave background to the spatial distribution of galaxies. This model has a few free parameters representing fundament quantities, like the geometry and expansion rate of the Universe, the amount and nature of dark energy, and the sum of neutrino masses. Knowing the value of these parameters will improve our knowledge on the fundamental constituents and laws governing our universe. Thus, one of most important goals of modern cosmology is to constrain the value of these parameters with the highest accuracy. Therefore, as an extrapolation between the ideas of the standard cosmostatistics and the use of machine learning techniques will improve even better the constrain of the cosmological parameters without to be worried about the intrinsic uncertainties of the data [100].

\section{Acknowledgments}

CE-R acknowledges the Royal Astronomical Society as FRAS 10147 and supported by PAPIIT Project IA100220 and ICN-UNAM projects.

\section{References}

[1] Weinberg, S. The Cosmological Constant Problems. 2000, arXiv:astro-ph/0005265.

[2] Sahni, V.; Starobinsky, A.A. The Case for a positive cosmological Lambda term. Int. J. Mod. Phys. D 2000, 9, 373-443.

[3] Feng, L.; Lu,T. A new equation of state for dark energy model. J. Cosmol. Astropart. Phys. 2011, $2011,034$.

[4] Stefancic, H. Equation of state description of the dark energy transition between quintessence and phantom regimes. J. Phys. Conf. Ser. 2006, 39, 182.

[5] Wang, Y.; Tegmark, M. Uncorrelated measurements of the cosmic expansion history and dark energy from supernovae. Phys. Rev. D 2005, 71, 103513.

[6] Barboza, E.M.; Alcaniz, J.S.; Zhu, Z.-H.; Silva, R. A generalized equation of state for dark energy. Phys. Rev. D 2009, 80, 043521.

[7] Pantazis, G.; Nesseris, S.; Perivolaropoulos, L. Comparison of thawing and freezing dark energy parametrizations. Phys. Rev. D 2016, 93, 103503.

[8] Wang, Y. Figure of merit for dark energy constraints from current observational data. Phys. Rev. D $2008,77,123525$.

[9] Jassal, H.K.; Bagla, J.S.; Padmanabhan, T. WMAP constraints on low redshift evolution of dark energy. Mon. Not. Roy. Astron. Soc. 2005, 356, L11.

[10] Ade, P.A.R.; Aghanim, N.; Arnaud, M.; Ashdown, M.; Aumont, J.; Baccigalupi, C.; Banday, A.J.; Barreiro, R.B.; Bartlett, J.G.; Bartolo, N.; et al. Planck 2015 Results. XIII. Cosmological Parameters. 2015, arXiv:astro-ph.CO/1502.01589. 
[11] Betoule, M.; Kessler, R.; Guy, J.; Mosher, J.; Hardin, D.; Biswas, R.; Astier, P.; El-Hage, P.; Konig, M.; Kuhlmann, S.; et al. Improved cosmological constraints from a joint analysis of the SDSS-II and SNLS supernova samples. Astron. Astrophys. 2014, 568, A22.

[12] Busca, N.G.; Delubac, T.; Rich, J.; Bailey, S.; Font-Ribera, A.; Kirkby, D.; Le Goff, J.-M.; Pieri, M.M.; Slosar, A.; Aubourg, É.; et al. Baryon Acoustic Oscillations in the Ly- $\alpha$ forest of BOSS quasars. Astron. Astrophys. 2013, 552, A96.

[13] Lazkoz, R.; Nesseris S.; Perivolaropoulos, L. Exploring Cosmological Expansion Parametrizations with the Gold SnIa Dataset. J. Cosmol. Astropart. Phys. 2005 , 2005, 010.

[14] Peebles, P.J.E.; Ratra, B. The Cosmological constant and dark energy. Rev. Mod. Phys. 2003, 75, 559-606.

[15] Huterer, D.; Turner, M.S. Probing the dark energy: Methods and strategies. Phys. Rev. D 2001, 64, 123527.

[16] Weller, J.; Albrecht, A. Future supernovae observations as a probe of dark energy. Phys. Rev. D 2002, $65,103512$.

[17] Wang, F.Y.; Dai, Z.G. Constraining Dark Energy and Cosmological Transition Redshift with Type Ia Supernovae. Chin. J. Astron. Astrophys. 2006, 6, 561.

[18] Linder, E.V. The dynamics of quintessence, The quintessence of dynamics. Gen. Rel. Grav. 2008, 40, 329-356.

[19] Chevallier, M.; Polarski, D. Accelerating universes with scaling dark matter. Int. J. Mod. Phys. D 2001, 10, 213-223.

[20] Barboza, E.M., Jr.; Alcaniz, J.S. A parametric model for dark energy. Phys. Lett. B 2008, 666, 415-419.

[21] Wetterich, C. Phenomenological parameterization of quintessence. Phys. Lett. B 2004, 594, $17-22$.

[22] Wetterich, C. Cosmology with Varying Scales and Couplings. 2003, arXiv:hep-ph/0302116.

[23] Escamilla-Rivera, C.; Casarini, L.; Fabris, J.C.; Alcaniz, J.S. Linear and non-linear perturbations in dark energy models. 2016, arXiv:1605.01475.

[24] Conley, A.; Guy, J.; Sullivan, M.; Regnault, N.; Astier, P.; Balland, C.; Basa, S.; Carlberg, R.G.; Fouchez, D.; Hardin, D.; et al. Supernova Constraints and Systematic Uncertainties from the First 3 Years of the Supernova Legacy Survey. Astrophys. J. Suppl. 2011, 192, 1.

[25] Beutler, F.; Blake, C.; Colless, M.; Jones, D.H.; Staveley-Smith, L.; Campbell, L.; Parker, Q.; Saunders, W.; Watson, F.; et al. The 6dF Galaxy Survey: Baryon Acoustic Oscillations and the Local Hubble Constant. Mon. Not. Roy. Astron. Soc. 2011, 416, 3017-3032.

[26] Anderson, L.; Aubourg, É; Bailey, S.; Beutler, F.; Bhardwaj, V.; Blanton, M.; Bolton, A.S.; Brinkmann, J.; Brownstein, J.R.; Burden, A.; et al. The clustering of galaxies in the SDSS-III Baryon Oscillation Spectroscopic Survey: baryon acoustic oscillations in the Data Releases 10 and 11 Galaxy samples. Mon. Not. Roy. Astron. Soc. 2014, 441, 24-62.

[27] Xu, X.; Padmanabhan, N.; Eisenstein, D.J.; Mehta, K.T.; Cuesta, A.J. A $2 \%$ Distance to $z=0.35$ by reconstructing baryon acoustic oscillations-II: Fitting techniques. Mon. Not. Roy. Astron. Soc. 2012, 427, 2146-2167.

[28] Blake, C.; Brough, S.; Colless, M.; Contreras, C.; Couch, W.; Croom, S.; Croton, D.; Davis, T.M.; Drinkwater, M.J.; Forster, K.; et al. The WiggleZ Dark Energy Survey: Joint measurements of the expansion and growth history at z < 1. Mon. Not. Roy. Astron. Soc. 2012, 425, 405-414. 
[29] Press W.H.; Teukolsky A.; Vetterling W.; Flannery B. Numerical Recipes, 3rd, ed.; Cambridge Press: New York, USA, 1994.

[30] Escamilla-Rivera, C.; Lazkoz, R.; Salzano, V.; Sendra, I. Tension between SN and BAO: Current status and future forecasts. J. Cosmol. Astropart. Phys. 2011, doi:10.1088/1475-7516/2011/09/003.

[31] Burigana, C.; Destri, C.; de Vega, H.J.; Gruppuso, A.; Mandolesi, N.; Natoli, P.; Sanchez, N.G. Forecast for the Planck precision on the tensor to scalar ratio and other cosmological parameters. Astrophys. J. 2010, 724, 588.

[32] Bayes, R.T. An essay toward solving a problem in the doctrine of chances. Phil. Trans. R. Soc. Lond. 1764, 53, 370-418.

[33] Gregory, P. Bayesian Logical Data Analysis for the Physical Sciences; Cambridge University Press: New York, USA, 2005.

[34] Trotta, R. Applications of Bayesian model selection to cosmological parameters. Mon. Not. Roy. Astron. Soc. 2007, 378, 72-82.

[35] Skilling J. Bayesian annal 1, 2006, 833.

http://www.mrao.cam.ac.uk/ steve/maxent2009/images/skilling.pdf

[36] Liddle, A.R.; Mukherjee, P.; Parkinson, D.; Wang, Y. Present and future evidence for evolving dark energy. Phys. Rev. D 2006, 74, 123506.

[37] Jeffreys, H. Theory of Probability, 3rd ed.; Oxford University Press: Oxford, United Kingdom. 1998.

[38] Albrecht, A.; Amendola, L.; Bernstein, G.; Clowe, D.; Eisenstein, D.; Guzzo, L.; Hirata, C.; Huterer, D.; Kirshner, R.; Kolb, E.; et al. Findings of the Joint Dark Energy Mission Figure of Merit Science Working Group. 2009, arXiv:0901.0721

[39] Liddle, A.R. How many cosmological parameters? Mon. Not. Roy. Astron. Soc. 2004, 351, L49-L53.

[40] A. G. Riess et al. [Supernova Search Team], Astron. J. 116, 1009 (1998) doi:10.1086/300499 [astro-ph/9805201].

[41] S. Perlmutter et al. [Supernova Cosmology Project Collaboration], Astrophys. J. 517, 565 (1999) doi:10.1086/307221 [astro-ph/9812133].

[42] http://desi.lbl.gov/

[43] https://www.darkenergysurvey.org/

[44] https://www. Isst.org/

[45] https://wfirst.gsfc.nasa.gov/

[46] M. Takada and B. Jain, Mon. Not. Roy. Astron. Soc. 340, 580 (2003) doi:10.1046/j.1365-8711.2003.06321.x [astro-ph/0209167].

[47] F. A. Marin et al. [WiggleZ Collaboration], Mon. Not. Roy. Astron. Soc. 432, 2654 (2013) doi:10.1093/mnras/stt520 [arXiv:1303.6644 [astro-ph.CO]].

[48] S. Tsujikawa, doi:10.1007/978-90-481-8685-38 arXiv:1004.1493 [astro-ph.CO].

[49] D. Huterer and M. S. Turner, Phys. Rev. D 64, 123527 (2001) doi:10.1103/PhysRevD.64.123527 [astro-ph/0012510].

[50] Y. Wang, Phys. Rev. D 77, 123525 (2008) doi:10.1103/PhysRevD.77.123525 [arXiv:0803.4295 [astro-ph]]. 
[51] C. Escamilla-Rivera, R. Lazkoz, V. Salzano and I. Sendra, JCAP 1109, 003 (2011) doi:10.1088/1475-7516/2011/09/003 [arXiv:1103.2386 [astro-ph.CO]].

[52] P. Bull et al., Phys. Dark Univ. 12, 56 (2016) doi:10.1016/j.dark.2016.02.001 [arXiv:1512.05356 [astro-ph.CO]].

[53] N. Aghanim et al. [Planck Collaboration], arXiv:1807.06209 [astro-ph.CO].

[54] L. Verde, T. Treu and A. G. Riess, arXiv:1907.10625 [astro-ph.CO].

[55] B. Ratra and P. J. E. Peebles, Phys. Rev. D 37, 3406 (1988). doi:10.1103/PhysRevD.37.3406

[56] C. Armendariz-Picon, V. F. Mukhanov and P. J. Steinhardt, Phys. Rev. Lett. 85, 4438 (2000) doi:10.1103/PhysRevLett.85.4438 [astro-ph/0004134].

[57] I. Sendra and R. Lazkoz, Mon. Not. Roy. Astron. Soc. 422, 776 (2012) doi:10.1111/j.1365-2966.2012.20661.x [arXiv:1105.4943 [astro-ph.CO]].

[58] G. B. Zhao, D. Bacon, R. Maartens, M. Santos and A. Raccanelli, arXiv:1501.03840 [astro-ph.CO].

[59] C. Escamilla-Rivera, Galaxies 4, no. 3, 8 (2016) doi:10.3390/galaxies4030008 [arXiv:1605.02702 [astro-ph.CO]].

[60] M. Rezaei, M. Malekjani, S. Basilakos, A. Mehrabi and D. F. Mota, Astrophys. J. 843, no. 1, 65 (2017) doi:10.3847/1538-4357/aa7898 [arXiv:1706.02537 [astro-ph.CO]].

[61] C. Escamilla-Rivera and S. Capozziello, International Journal of Modern Physics D (2019) doi:10.1142/S0218271819501542 [arXiv:1905.04602 [gr-qc]].

[62] L. G. Jaime, L. Patiño and M. Salgado, Phys. Rev. D 89, no. 8, 084010 (2014) doi:10.1103/PhysRevD.89.084010 [arXiv:1312.5428 [gr-qc]].

[63] R. Lazkoz, M. Ortiz-Baños and V. Salzano, Eur. Phys. J. C 78, no. 3, 213 (2018) doi:10.1140/epjc/s10052-018-5711-6 [arXiv:1803.05638 [astro-ph.CO]].

[64] S. Capozziello, R. D’ Agostino and O. Luongo, Int. J. Mod. Phys. D 28, no. 10, 1930016 (2019) doi:10.1142/S0218271819300167 [arXiv:1904.01427 [gr-qc]].

[65] J. Alberto Vazquez, M. Bridges, M. P. Hobson and A. N. Lasenby, JCAP 1209, 020 (2012) doi:10.1088/1475-7516/2012/09/020 [arXiv:1205.0847 [astro-ph.CO]].

[66] Marina Seikel, Chris Clarkson, Mathew Smith JCAP06(2012)036 doi: 10.1088/1475-7516/2012/06/036 [arXiv:1204.2832]

[67] A. Montiel, R. Lazkoz, I. Sendra, C. Escamilla-Rivera and V. Salzano, Phys. Rev. D 89, no. 4, 043007 (2014) doi:10.1103/PhysRevD.89.043007 [arXiv:1401.4188 [astro-ph.CO]].

[68] G. B. Zhao et al., Nat. Astron. 1, no. 9, 627 (2017) doi:10.1038/s41550-017-0216-z [arXiv:1701.08165 [astro-ph.CO]].

[69] L. G. Jaime, M. Jaber and C. Escamilla-Rivera, Phys. Rev. D 98, no. 8, 083530 (2018) doi:10.1103/PhysRevD.98.083530 [arXiv:1804.04284 [astro-ph.CO]].

[70] Geron Aurelien. Hands-On Machine Learning with Scikit-Learn and Tensorflow: Concepts, Tools, and Techniques to Build Intelligent Systems. ISBN-10: 1491962291. O’Reilly Media. 2017.

[71] T. Charnock and A. Moss, Astrophys. J. 837, no. 2, L28 (2017) doi:10.3847/2041-8213/aa603d [arXiv:1606.07442 [astro-ph.IM]].

[72] A. Mathuriya et al., arXiv:1808.04728 [astro-ph.CO]. 
[73] R. Kessler, A. Conley, S. Jha and S. Kuhlmann, arXiv:1001.5210 [astro-ph.IM].

[74] A. Moss, arXiv:1810.06441 [astro-ph.IM].

[75] A. Moss, arXiv:1903.10860 [astro-ph.CO].

[76] C. Escamilla-Rivera, M. A. C. Quintero and S. Capozziello, arXiv:1910.02788 [astro-ph.CO].

[77] Stefancic, H. Equation of state description of the dark energy transition between quintessence and phantom regimes. J. Phys. Conf. Ser. 2006, 39, 182.

[78] Lazkoz, R.; Nesseris S.; Perivolaropoulos, L. Exploring Cosmological Expansion Parametrizations with the Gold SnIa Dataset. J. Cosmol. Astropart. Phys. 2005 , 2005, 010.

[79] Peebles, P.J.E.; Ratra, B. The Cosmological constant and dark energy. Rev. Mod. Phys. 2003, 75, 559-606.

[80] Chevallier, M.; Polarski, D. Accelerating universes with scaling dark matter. Int. J. Mod. Phys. D 2001, 10, 213-223.

[81] Linder, E.V. The dynamics of quintessence, The quintessence of dynamics. Gen. Rel. Grav. 2008, 40, 329-356.

[82] B. C. Paul and P. Thakur, JCAP 1311, 052 (2013) doi:10.1088/1475-7516/2013/11/052 [arXiv:1306.4808 [astro-ph.CO]].

[83] D. M. Scolnic et al., Astrophys. J. 859 (2018) 101

[84] M. Betoule et al. [SDSS Collaboration], Astron. Astrophys. 568, A22 (2014) doi:10.1051/0004-6361/201423413 [arXiv:1401.4064 [astro-ph.CO]].

[85] M. Ntampaka et al., arXiv:1902.10159 [astro-ph.IM].

[86] J. Schmelzle, A. Lucchi, T. Kacprzak, A. Amara, R. Sgier, A. Réfrégier and T. Hofmann, arXiv:1707.05167 [astro-ph.CO].

[87] S. Ruder. arXiv:1609.04747

[88] Anais Moller and Thibault de Boissiere, arXiv:1901.06384

[89] A. Géron Hands-On Machine Learning with Scikit-Learn \& TensorFlow.2017 O’REILLY.

[90] C. Escamilla-Rivera and J. C. Fabris, Galaxies 4, no. 4, 76 (2016) doi:10.3390/galaxies4040076 [arXiv:1511.07066 [astro-ph.CO]].

[91] C. Escamilla-Rivera, L. Casarini, J. C. Fabris and J. S. Alcaniz, JCAP 1611, 010 (2016) doi:10.1088/1475-7516/2016/11/010 [arXiv:1605.01475 [astro-ph.CO]].

[92] I. Goodfellow, Y. Bengio and A. Courville Deep Learning. MIT Press.2016. http://www. deeplearningbook.org

[93] Wojciech Zaremba, Ilya Sutskever. arXiv:1505.00521 [cs.LG]

[94] C. Escamilla-Rivera, Galaxies 4, no. 3, 8 (2016) doi:10.3390/galaxies4030008 [arXiv:1605.02702 [astro-ph.CO]].

[95] D. Pedamonti. arXiv:1804.02763 [cs.LG]

[96] Y. Gal \& Z. Ghahramani. A Theoretically Grounded Application of Dropout in Recurrent Neural Networks.2016 [arXiv:1512.05287v5] 
[97] G. Bárcenas-Enríquez, C. Escamilla-Rivera and M. A. Garcia-Aspeitia, Rev. Mex. Fis. 64, no. 6, 584 (2018) doi:10.31349/RevMexFis.64.584 [arXiv:1803.03283 [gr-qc]].

[98] L. G. Jaime, M. Jaber and C. Escamilla-Rivera, Phys. Rev. D 98, no. 8, 083530 (2018) doi:10.1103/PhysRevD.98.083530 [arXiv:1804.04284 [astro-ph.CO]].

[99] C. Escamilla-Rivera and S. Capozziello, Int. J. Mod. Phys. D 28, no. 12, 1950154 (2019) doi:10.1142/S0218271819501542 [arXiv:1905.04602 [gr-qc]].

[100] C. Escamilla-Rivera, M. A. C. Quintero and S. Capozziello, arXiv:1910.02788 [astro-ph.CO]. 